\title{
Clinical Study \\ Clinical Outcomes after Uncomplicated Cataract Surgery with Implantation of the Tecnis Toric Intraocular Lens
}

\author{
Wojciech Lubiński, Beata Kaźmierczak, Jolanta Gronkowska-Serafin, \\ and Karolina Podborączyńska-Jodko
}

Clinic of Ophthalmology, Pomeranian Medical University, 70-111 Szczecin, Poland

Correspondence should be addressed to Wojciech Lubiński; lubinski@pro.onet.pl

Received 20 November 2015; Revised 29 January 2016; Accepted 2 February 2016

Academic Editor: Lisa Toto

Copyright (C) 2016 Wojciech Lubiński et al. This is an open access article distributed under the Creative Commons Attribution License, which permits unrestricted use, distribution, and reproduction in any medium, provided the original work is properly cited.

Purpose. To evaluate the clinical outcomes after uncomplicated cataract surgery with implantation of an aspheric toric intraocular lens (IOL) during a 6-month follow-up. Methods. Prospective study including 27 consecutive eyes of 18 patients (mean age: $66.1 \pm$ 11.4 years) with a visually significant cataract and corneal astigmatism $\geq 0.75 \mathrm{D}$ and undergoing uncomplicated cataract surgery with implantation of the Tecnis ZCT toric IOL (Abbott Medical Optics). Visual, refractive, and keratometric outcomes as well as IOL rotation were evaluated during a 6-month follow-up. At the end of the follow-up, patient satisfaction and perception of optical/visual disturbances were also evaluated using a subjective questionnaire. Results. At 6 months after surgery, mean LogMAR uncorrected (UDVA) and corrected distance visual acuity (CDVA) were $0.19 \pm 0.12$ and $0.14 \pm 0.10$, respectively. Postoperative UDVA of $20 / 40$ or better was achieved in $92.6 \%$ of eyes. Mean refractive cylinder decreased significantly from $-3.73 \pm 1.96$ to $-1.42 \pm 0.88 \mathrm{D}(p<0.001)$, while keratometric cylinder did not change significantly $(p=0.44)$. Mean absolute IOL rotation was $1.1 \pm 2.4^{\circ}$, with values of more than $5^{\circ}$ in only 2 eyes $(6.9 \%)$. Mean patient satisfaction score was $9.70 \pm 0.46$, using a scale from 0 (not at all satisfied) to 10 (very satisfied). No postoperative optical/visual disturbances were reported. Conclusion. Cataract surgery with implantation of the Tecnis toric IOL is an effective method of refractive correction in eyes with corneal astigmatism due to the good IOL positional stability, providing high levels of patient's satisfaction.

\section{Introduction}

Approximately $60 \%$ of patients undergoing cataract surgery have more than $0.75 \mathrm{D}$ of corneal astigmatism [1]. If uncorrected, this astigmatism results in reduced visual acuity and increased spectacle dependence in pseudophakic eyes [2]. The correction of corneal astigmatism in cataract surgery can be achieved using different surgical techniques (corneal or limbal relaxing incisions, modification of the placement of the incision site) $[3,4]$ or by implanting a toric intraocular lens (IOL) [5]. Several studies have reported successful visual and refractive outcomes after implantation of different models of toric IOL [5-19]. The Tecnis ZCT toric IOL combines an aspheric profile with a toric optic. To this date, only few clinical results with this specific type of toric IOL have been published, several with a rather short follow-up of less than 6 months $[9,13,15,16,19]$. The purpose of the current study was to report our clinical outcomes at 6 months after uncomplicated cataract surgery with implantation of the Tecnis ZCT toric IOL.

\section{Methods}

2.1. Patients. This nonrandomized prospective case series included 27 eyes of 18 patients undergoing cataract surgery with implantation of the Tecnis ZCT toric IOL (Abbott Medical Optics Inc.). Inclusion criteria were visually significant cataract, age of 18 years or older, and preoperative corneal astigmatism of $0.75 \mathrm{D}$ or higher. Patients were excluded from the study when the following conditions were present: potential visual acuity of less than $0.2 \operatorname{LogMAR}$ in each eye due to ocular pathological processes, systemic or ocular medication that could affect vision, any chronic or acute 
pathology that could alter the result, previous ocular surgery, amblyopia, strabismus, forme fruste or clinical keratoconus, pupil abnormalities, capsular or zonular abnormalities with the potential of inducing IOL decentration or tilting, and participation in another clinical study. The study adhered to the tenets of the Declaration of Helsinki and was approved by the local ethics committee.

2.2. Preoperative and Postoperative Evaluation. Before surgery, all patients underwent a complete ophthalmological examination that included the following: manifest refraction, measurement of LogMAR uncorrected (UDVA), and corrected distance visual acuity (CDVA), biometry and keratometry with the IOLMaster partial coherence interferometry device (Carl Zeiss Meditec AG), corneal topography to exclude irregular astigmatism, slit lamp examination, and dilated funduscopy. The IOL manufacturer's web-based toric calculator was used to determine the required cylinder power and axis for the IOL that was going to be implanted. The preferred clear corneal incision location was the superior temporal quadrant and the surgeon's estimated surgically induced corneal astigmatism was $0.75 \mathrm{D}$.

On the first day after surgery, the axis position of the implanted toric IOL was analyzed under pupil dilation $(1.0 \%$ tropicamide) with the slit lamp by performing a thin coaxial slit rotation until it overlapped the axis margins on the IOL. In two cases of a rotation of the IOL axis of more than 5 degrees the IOL was repositioned in the operating room and were excluded from study. Six months after surgery, manifest refraction, LogMAR UDVA and CDVA, and corneal astigmatism were measured. Patients were asked about the incidence of postoperative optical/visual disturbances, such as arc of light, halos, ghosting, or glare, and about their satisfaction with the achieved visual outcome, using a scale from 0 to $10(0=$ not at all satisfied, $10=$ very satisfied $)$.

2.3. Intraocular Lens. The 1-piece aspheric toric IOL Tecnis ZCT has $6.0 \mathrm{~mm}$ optic diameter and an overall length of $13.0 \mathrm{~mm}$. It has a 360-degree square edge with frosting to reduce migration of lens epithelial cells and possible glare effects. The C-loop haptics are aimed at providing a 3point fixation in the capsular bag for maintaining good IOL centration and rotational stability. The lens is made of a hydrophobic acrylic material with a high Abbe value (55) which reduces the level of longitudinal chromatic aberration with the potential of improving contrast sensitivity [20].

2.4. Surgical Technique. Before surgery, after instilling topical anesthetic eye drops and with the patient in supine position, the corneal limbus was marked at the $0^{\circ}, 90^{\circ}$, and $180^{\circ}$ meridian using the toric reference marker AE 2791 (Asico). Intraoperatively the required IOL axis was determined with the aid of the axis marker AE 2794 (Asico). After phacoemulsification, the IOL was inserted into the capsular bag using the Unfolder Platinum 1 system (Abbott Medical Optics Inc.) through a $2.2 \mathrm{~mm}$ corneal incision in the superotemporal quadrant. After the removal of the ophthalmic viscosurgical
TABLE 1: Patient demographics and preoperative data in the analyzed sample.

\begin{tabular}{lc}
\hline Parameter & Value \\
\hline Age (years) & $66.1 \pm 11.4$ \\
Mean \pm SD & 37 to 79 \\
Range & \\
Sex (\%) & $6(33 \%)$ \\
Male & $12(67 \%)$ \\
Female & \\
Sphere (D) & $-2.70 \pm 6.70$ \\
Mean \pm SD & -18.50 to 5.50 \\
Range & \\
Cylinder (D) & $-3.73 \pm 1.96$ \\
Mean \pm SD & -8.50 to -1.50 \\
Range & \\
Keratometry (D) & $42.58 \pm 1.61$ \\
K1 (steep) & $45.77 \pm 1.82$ \\
K2 (flat) & \\
Axial length (mm) & $23.87 \pm 1.38$ \\
Mean \pm SD & 22.19 to 27.83 \\
Range & \\
Mean IOL power (D) & $19.59 \pm 4.58$ \\
Sphere & $-3.64 \pm 0.54$ \\
Cylinder & \\
\hline
\end{tabular}

device (Discovisc, Alcon) from the capsular bag, the IOL was rotated, if necessary, to the correct axis position.

2.5. Data Analysis. Distribution of analyzed data was performed using the Kolmogorov-Smirnov test. All data presented in the current study were not normally distributed and therefore nonparametric statistics were used. The Wilcoxon ranked sum test was used to compare changes in visual and refractive parameters between preoperative and postoperative examinations, considering a significance level of $p<$ 0.05 .

The spherocylindrical refractions obtained before and after surgery were converted to vectorial notation using the power vector method described by Thibos and Horner [21]. According to the power vector method, manifest refractions in conventional script notation ( $S$ [sphere], $C$ [cylinder $] \times \varphi$ [axis]) were converted to power vector coordinates and overall blurring strength $(B)$ by the following formulas: $M=S+C / 2 ; J_{0}=(-C / 2) \cos (2 \varphi) ; J_{45}=(-C / 2) \sin (2 \varphi) ;$ and $B=\left(M^{2}+J_{0}^{2}+J_{45}^{2}\right)^{1 / 2}$.

\section{Results}

Twenty seven eyes of 18 patients were enrolled in the study. Table 1 summarizes the patient demographics and the preoperative data.

3.1. Visual and Refractive Outcomes. Table 2 shows the preoperative and 6-month postoperative visual and refractive 
TABLE 2: Preoperative and 6-month postoperative visual and refractive outcomes.

\begin{tabular}{lccc}
\hline Parameter $($ mean \pm SD) & Preoperative & 6 months postoperative & $p$ value \\
\hline LogMAR UDVA & $0.78 \pm 0.22$ & $0.19 \pm 0.12$ & $<0.001^{*}$ \\
LogMAR CDVA & $0.49 \pm 0.39$ & $0.14 \pm 0.10$ & \\
Manifest refraction & & & $<0.001^{*}$ \\
$\quad$ Sphere (D) & $-2.70 \pm 6.70$ & $0.62 \pm 0.58$ & $0.11^{*}$ \\
Cylinder (D) & $-3.73 \pm 1.96$ & $-1.42 \pm 0.88$ & $<0.001^{*}$ \\
$\quad$ Spherical equivalent (D) & $-4.63 \pm 7.24$ & $0.13 \pm 0.43$ & $0.01^{*}$ \\
Keratometric cylinder (D) & $-3.19 \pm 1.59$ & $-3.16 \pm 1.44$ & 0.44 \\
\hline
\end{tabular}

*Value statistically significant.

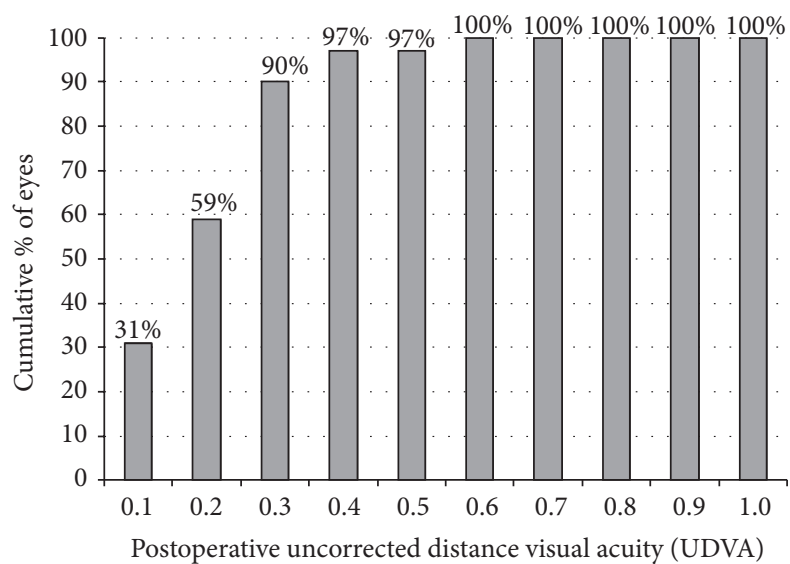

FIGURE 1: Distribution of the postoperative uncorrected distance visual acuity (UDVA) LogMAR.

outcomes in the analyzed sample. A significant improvement was found after surgery in LogMAR UDVA and CDVA $(p<0.001)$. At 6 months postoperatively, a LogMAR UDVA of 0.30 (20/40 Snellen) or better was achieved in 25 eyes (92.6\%) (Figure 1). Postoperative CDVA was 0.30 or better (20/40 Snellen) in all eyes $(100 \%)$ and $0.00 \operatorname{logMAR}$ $(20 / 20$ Snellen) in 9 eyes (33.33\%). All eyes (100\%) showed a mean spherical equivalent within $\pm 1.00 \mathrm{D}$ of emmetropia. The refractive cylinder decreased significantly after surgery $(p<0.001)$ while the keratometric cylinder did not change significantly $(p=0.44)$. Figure 2 shows the distribution of preoperative and postoperative $J_{0}$ and $J_{45}$ refractive cylinder vectors. Postoperatively, the data points concentrate around the origin $(0,0)$ whereas preoperatively the data showed a high level of scattering.

3.2. Intraocular Lens Alignment. One day after surgery two eyes required additional repositioning surgery. Mean absolute IOL misalignment at 6 month after surgery was $1.1 \pm$ 2.4 degrees (range, 0 to 8 degrees). Two eyes (7.4\%) had an IOL misalignment of more than 5 degrees (8 degrees) and 4 eyes (17.2\%) showed a misalignment of less than 5 degrees. Accurate alignment of the IOL with its intended axis was obtained in more than half of the operated eyes $(19 / 27$, $70.37 \%)$.

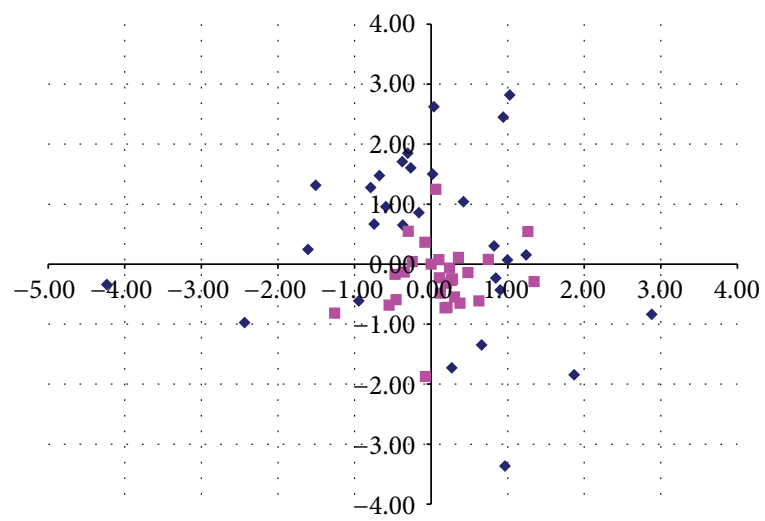

- Before $J_{0}$ before $J_{45}$

- After $J_{0}$ after $J_{45}$

FIGURE 2: Distribution of the preoperative and postoperative $J_{0}$ and $J_{45}$ vectors representing the refractive astigmatism.

3.3. Patient Satisfaction and Visual Disturbances. At 6 months after surgery, mean patient satisfaction score was $9.70 \pm 0.47$ using a scale from 0 (not at all satisfied) to 10 (very satisfied). Specifically, 5 patients scored their satisfaction as 9 and the rest as 10. No optical or visual disturbances were reported by any of the patients from the analyzed sample.

3.4. Complications. There were no intraoperative complications. In one eye, a retinal detachment occurred at 2 months after surgery that was successfully treated by pars plana vitrectomy.

\section{Discussion}

Recently, the correction of corneal astigmatism in cataract surgery by implanting toric IOLs has gained popularity due to the increased patient demands and the excellent clinical outcomes reported with these IOLs [5]. In the current study we evaluated a specific modality of aspheric toric IOL, allowing the correction of a great variety of corneal astigmatism as it is available in cylinder powers of 1.00, 1.50, $2.25,3.00$, and $4.00 \mathrm{D}$ (equivalent to $0.69,1.03,1.54,2.06$, and $2.74 \mathrm{D}$ at the corneal plane, resp.). Some cases of higher 
corneal astigmatism which cannot be completely controlled with the available cylinder powers of this IOL model have nevertheless been included in our series. In general, good visual and refractive outcomes have been obtained with the Tecnis toric IOL, mainly due to its good positional stability.

Our results confirmed the results of previous studies evaluating the same IOL and demonstrating its ability as an effective method of corneal astigmatism reduction $[9,13,15$, $16,19]$. Specifically, we found a mean reduction in refractive astigmatism of $2.31 \mathrm{D}$ that was statistically significant. At 6 months after surgery, refractive astigmatism ranged from 0.00 to $-3.75 \mathrm{D}$, with a mean value of $-1.42 \pm 0.88 \mathrm{D}$. Lower postoperative refractive cylinder values have been reported by other authors evaluating the same type of toric IOL [9, $13,15,16,19]$. Waltz et al. [9] found a mean percentage of refractive cylinder reduction of $76.27 \pm 33.09 \%$ at 6 months after cataract surgery with implantation of the Tecnis toric IOL, but in a group of eyes only requiring cylinder correction of 0.75 to $1.50 \mathrm{D}$. In our sample, twelve eyes (37.04\%) had a preoperative corneal astigmatism of more than $-3.00 \mathrm{D}$ in which corneal astigmatism was not corrected completely but reduced significantly. Iovieno et al. [22] obtained a mean postoperative refractive cylinder of $-1.81 \pm$ $1.10 \mathrm{D}$ in a group of eyes with high corneal astigmatism (preoperative refractive cylinder: $-4.72 \pm 1.13 \mathrm{D}$ ) undergoing cataract surgery with implantation of a custom-made highpower toric IOL. Cervantes-Coste et al. [23] found a residual refractive cylinder of $0.55 \pm 0.60 \mathrm{D}$ at 3 months after cataract surgery with implantation of a toric IOL in 19 eyes with symmetric corneal astigmatism of more than $2.25 \mathrm{D}$. Ouchi and Kinoshita [24] found similar results in another sample of eyes undergoing cataract surgery with implantation of a toric IOL for the correction of corneal astigmatism of more than $2.50 \mathrm{D}$ (mean postoperative refractive cylinder: $1.07 \pm 0.60 \mathrm{D}$ ). Some authors have even reported the necessity of implanting two IOLs in piggyback for achieving an acceptable refractive outcome in eyes with high corneal astigmatism [25]. The toric IOL evaluated in our sample was able to provide an effective correction of corneal astigmatism, even in cases requiring high levels of correction, reaching a mean percentage of refractive cylinder reduction of $61.93 \pm 18.4 \%$. All eyes $(100 \%)$ had a mean postoperative spherical equivalent within $\pm 1.00 \mathrm{D}$. This is consistent with the results of previous studies evaluating the same toric IOL [13] and also other modalities of toric IOLs $[5,11,12]$.

In agreement with the good refractive outcomes, excellent UDVA results were obtained which was the main reason for the high levels of postoperative patient satisfaction. Mean LogMAR UDVA was 0.19 with all eyes achieving a value of 0.30 LogMAR or better, which is an outcome comparable or even better than that reported by other authors investigating toric IOLs [6-17, 26, 27]. Sheppard et al. [15] reported that $88 \%$ of eyes achieved a UDVA of $20 / 40$ or better after implantation of the same toric IOL as evaluated in our series. Similarly, Ferreira and Almeida [16] found a postoperative UDVA of $0.3 \log$ MAR or better in $100 \%$ of eyes implanted with the same toric IOL, whereas Mazzini [13] found that postoperative UDVA was $0.1 \operatorname{LogMAR}(20 / 25)$ or better in $94.74 \%$ of eyes. Alió et al. [26] reported a postoperative
UDVA of at least $20 / 40$ in $76 \%$ of eyes implanted with a specific modality of microincision toric IOL. Kersey et al. [27] reported a mean postoperative UDVA value of 0.1 LogMAR in a sample of eyes implanted with a specific type of toric IOL, with $93 \%$ of eyes achieving a value of 0.3 LogMAR or better. LogMAR CDVA was also excellent in our series, with a mean postoperative value of 0.13 .

Postoperative rotational stability of a toric IOL has a crucial influence on the final visual outcome. An undesirable postoperative IOL rotation may be the result of several factors, such as an incomplete removal of viscoelastics from the eye (reduced friction between the haptics and capsular bag with postoperative intraocular pressure changes) [28] or a postoperative significant capsule shrinkage. In the current study, mean IOL misalignment from the target axis was very small $\left(1.1 \pm 2.4^{\circ}\right.$, range, 0 to $\left.8^{\circ}\right)$, which is consistent with the good visual and refractive outcomes obtained. Other studies evaluating the same IOL have reported similar or slightly higher levels of IOL misalignment $[9,13,15,16,19]$. Ferreira and Almeida [16] found a mean toric IOL axis misalignment of $3.15^{\circ}$ in 20 eyes at 2 months after the implantation of the same toric IOL as used in our study, with no IOL rotating more than $10^{\circ}$. Similarly, Sheppard et al. [15] obtained 2 months postoperatively a mean IOL axis misalignment of $3.40^{\circ}$ in a sample of 67 eyes, with only one eye showing a rotation of more than $10^{\circ}$. At 6 months after surgery, Mazzini [13] found a mean IOL misalignment of $3.33^{\circ}$ in a sample of 19 consecutive eyes, with none of the eyes showing an IOL rotation of more than $7^{\circ}$. Hirnschall et al. [19] and Waltz et al. [9] obtained mean IOL misalignments of $3.6^{\circ}$ and $1.89 \pm 2.27^{\circ}$ for the same toric IOL, respectively. Compared to other models of toric IOLs, our results were similar or better $[6,7,10,11,14,26,27]$. A mean IOL misalignment of $7.67 \pm 4.04^{\circ}$ was reported by Lam et al. [7] in a study evaluating a group of eyes implanted with a specific type of toric IOL. Miyake and coauthors [10] observed a mean IOL rotation of $4.5 \pm 4.9^{\circ}$ within 1 day postoperatively in a group of eyes implanted with a specific modality of aspheric toric IOL (Acrysof IQ toric SN6AT). These authors found that the rotation was more than $20^{\circ}$ in 6 eyes, all of which had an axial length of more than $25.0 \mathrm{~mm}$, with all rotations occurring within 10 days postoperatively [10]. Therefore, the aspheric toric IOL evaluated in the current study provided an excellent rotational stability which seemed to be mainly related to the IOL material (hydrophobic acrylic) [29] and design (3-point fixation system, offset haptics) [30].

Finally, patient satisfaction was high, with all patients scoring the visual outcome with a value of 9 or 10 in a scale from 0 to 10 postoperatively. Ahmed et al. [31] found in a group of patients implanted with a specific type of toric IOL that satisfaction with vision was rated 7 or higher by $94 \%$ of patients using a similar scale. These same authors found that the frequency and severity of halos and glare were significantly reduced pre- to postoperatively $[31,32]$. In our sample, no postoperative optical/visual disturbances were reported by any patient, which is consistent with the optical quality outcomes reported by other authors with this type of toric IOL $[13,16]$. 
In conclusion, cataract surgery with implantation of the Tecnis ZCT toric IOL provides an effective and predictable refractive correction in eyes with low to high levels of preexisting corneal astigmatism, providing high levels of visual quality and patient satisfaction. This might be due to the excellent rotational behavior of the IOL. Future studies should be conducted in order to evaluate the long term clinical outcomes with this modality of aspheric toric IOL.

\section{Conflict of Interests}

The authors have no proprietary or commercial interest in the medical devices that are involved in this paper.

\section{References}

[1] P. C. Hoffmann and W. W. Hütz, "Analysis of biometry and prevalence data for corneal astigmatism in 23239 eyes," Journal of Cataract and Refractive Surgery, vol. 36, no. 9, pp. 1479-1485, 2010.

[2] J. S. Wolffsohn, G. Bhogal, and S. Shah, "Effect of uncorrected astigmatism on vision," Journal of Cataract and Refractive Surgery, vol. 37, no. 3, pp. 454-460, 2011.

[3] L. D. Nichamin, "Astigmatism control," Ophthalmology Clinics of North America, vol. 19, no. 4, pp. 485-493, 2006.

[4] M. J. Carvalho, S. H. Suzuki, L. L. Freitas, B. C. Branco, P. Schor, and A. L. H. Lima, "Limbal relaxing incisions to correct corneal astigmatism during phacoemulsification," Journal of Refractive Surgery, vol. 23, no. 5, pp. 499-504, 2007.

[5] N. Visser, N. J. C. Bauer, and R. M. M. A. Nuijts, "Toric intraocular lenses: historical overview, patient selection, IOL calculation, surgical techniques, clinical outcomes, and complications," Journal of Cataract and Refractive Surgery, vol. 39, no. 4, pp. 624-627, 2013.

[6] A. Bachernegg, T. Rückl, C. Strohmaier, G. Jell, G. Grabner, and A. K. Dexl, "Vector analysis, rotational stability, and visual outcome after implantation of a new aspheric toric IOL," Journal of Refractive Surgery, vol. 31, no. 8, pp. 513-520, 2015.

[7] D. K. Lam, V. W. Chow, C. Ye, P. K. Ng, Z. Wang, and V. Jhanji, "Comparative evaluation of aspheric toric intraocular lens implantation and limbal relaxing incisions in eyes with cataracts and $\leq 3$ dioptres of astigmatism," British Journal of Ophthalmology, vol. 100, no. 2, pp. 258-262, 2016.

[8] E. M. Krall, E. M. Arlt, M. Hohensinn et al., "Vector analysis of astigmatism correction after toric intraocular lens implantation," Journal of Cataract \& Refractive Surgery, vol. 41, no. 4, pp. 790-799, 2015.

[9] K. L. Waltz, K. Featherstone, L. Tsai, and D. Trentacost, "Clinical outcomes of TECNIS toric intraocular lens implantation after cataract removal in patients with corneal astigmatism," Ophthalmology, vol. 122, no. 1, pp. 39-47, 2015.

[10] T. Miyake, K. Kamiya, R. Amano, Y. Iida, S. Tsunehiro, and K. Shimizu, "Long-term clinical outcomes of toric intraocular lens implantation in cataract cases with preexisting astigmatism," Journal of Cataract and Refractive Surgery, vol. 40, no. 10, pp. 1654-1660, 2014.

[11] B. V. Ventura, L. Wang, M. P. Weikert, S. B. Robinson, and D. D. Koch, "Surgical management of astigmatism with toric intraocular lenses," Arquivos Brasileiros de Oftalmologia, vol. 77, no. 2, pp. 125-131, 2014.
[12] E. H. Frieling-Reuss, "Comparative analysis of the visual and refractive outcomes of an aspheric diffractive intraocular lens with and without toricity," Journal of Cataract and Refractive Surgery, vol. 39, no. 10, pp. 1485-1493, 2013.

[13] C. Mazzini, "Visual and refractive outcomes after cataract surgery with implantation of a new toric intraocular lens," Case Reports in Ophthalmology, vol. 4, no. 2, pp. 48-56, 2013.

[14] L. Toto, L. Vecchiarino, E. D'Ugo et al., "Astigmatism correction with toric IOL: analysis of visual performance, position, and wavefront error," Journal of Refractive Surgery, vol. 29, no. 7, pp. 476-483, 2013.

[15] A. L. Sheppard, J. S. Wolffsohn, U. Bhatt et al., "Clinical outcomes after implantation of a new hydrophobic acrylic toric IOL during routine cataract surgery," Journal of Cataract and Refractive Surgery, vol. 39, no. 1, pp. 41-47, 2013.

[16] T. B. Ferreira and A. Almeida, "Comparison of the visual outcomes and OPD-scan results of AMO Tecnis toric and Alcon Acrysof IQ toric intraocular lenses," Journal of Refractive Surgery, vol. 28, no. 8, pp. 551-555, 2012.

[17] J. L. Alió, D. P. Piñero, J. Tomás, and A. Alesón, "Vector analysis of astigmatic changes after cataract surgery with toric intraocular lens implantation," Journal of Cataract and Refractive Surgery, vol. 37, no. 6, pp. 1038-1049, 2011.

[18] C. Pérez-Vives, T. Ferrer-Blasco, S. García-Lázaro, C. AlbarránDiego, and R. Montés-Micó, "Optical quality comparison between spherical and aspheric toric intraocular lenses," European Journal of Ophthalmology, vol. 24, no. 5, pp. 699-706, 2014.

[19] N. Hirnschall, S. Maedel, M. Weber, and O. Findl, "Rotational stability of a single-piece toric acrylic intraocular lens: a pilot study," American Journal of Ophthalmology, vol. 157, no. 2, pp. 405.el-411.el, 2014.

[20] H. Zhao and M. A. Mainster, "The effect of chromatic dispersion on pseudophakic optical performance," British Journal of Ophthalmology, vol. 91, no. 9, pp. 1225-1229, 2007.

[21] L. N. Thibos and D. Horner, "Power vector analysis of the optical outcome of refractive surgery," Journal of Cataract and Refractive Surgery, vol. 27, no. 1, pp. 80-85, 2001.

[22] A. Iovieno, S. N. Yeung, A. Lichtinger, M. Alangh, A. R. Slomovic, and D. S. Rootman, "Cataract surgery with toric intraocular lens for correction of high corneal astigmatism," Canadian Journal of Ophthalmology, vol. 48, no. 4, pp. 246-250, 2013.

[23] G. Cervantes-Coste, L. Garcia-Ramirez, E. Mendoza-Schuster, and C. Velasco-Barona, "High-cylinder acrylic toric intraocular lenses: a case series of eyes with cataracts and large amounts of corneal astigmatism," Journal of Refractive Surgery, vol. 28, no. 4, pp. 302-304, 2012.

[24] M. Ouchi and S. Kinoshita, "AcrySof IQ Toric IOL implantation combined with limbal relaxing incision during cataract surgery for eyes with astigmatism $>2.50$ D," Journal of Refractive Surgery, vol. 27, no. 9, pp. 643-647, 2011.

[25] J. P. Gills and M. A. Van der Karr, "Correcting high astigmatism with piggyback toric intraocular lens implantation," Journal of Cataract \& Refractive Surgery, vol. 28, no. 3, pp. 547-549, 2002.

[26] J. L. Alió, M. C. C. Agdeppa, V. C. Pongo, and B. El Kady, "Microincision cataract surgery with toric intraocular lens implantation for correcting moderate and high astigmatism: pilot study," Journal of Cataract and Refractive Surgery, vol. 36, no. 1, pp. 44-52, 2010.

[27] J. P. Kersey, A. O’Donnell, and C. D. Illingworth, "Cataract surgery with toric intraocular lenses can optimize uncorrected postoperative visual acuity in patients with marked corneal astigmatism," Cornea, vol. 26, no. 2, pp. 133-135, 2007. 
[28] F. A. S. Pereira, E. J. Milverton, and M. T. Coroneo, "MiyakeApple study of the rotational stability of the Acrysof toric intraocular lens after experimental eye trauma," Eye, vol. 24, no. 2, pp. 376-378, 2010.

[29] R. J. Linnola, M. Sund, R. Ylönen, and T. Pihlajaniemi, "Adhesion of soluble fibronectin, vitronectin, and collagen type IV to intraocular lens materials," Journal of Cataract and Refractive Surgery, vol. 29, no. 1, pp. 146-152, 2003.

[30] D. F. Chang, "Comparative rotational stability of single-piece open-loop acrylic and plate-haptic silicone toric intraocular lenses," Journal of Cataract and Refractive Surgery, vol. 34, no. 11, pp. 1842-1847, 2008.

[31] I. I. K. Ahmed, G. Rocha, A. R. Slomovic et al., "Visual function and patient experience after bilateral implantation of toric intraocular lenses," Journal of Cataract and Refractive Surgery, vol. 36, no. 4, pp. 609-616, 2010.

[32] J. Kwartz and K. Edwards, "Evaluation of the long-term rotational stability of single-piece, acrylic intraocular lenses," British Journal of Ophthalmology, vol. 94, no. 8, pp. 1003-1006, 2010. 


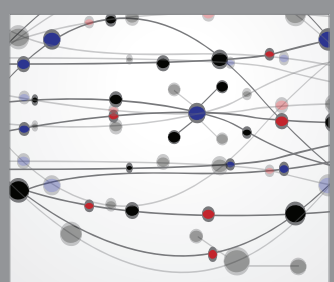

The Scientific World Journal
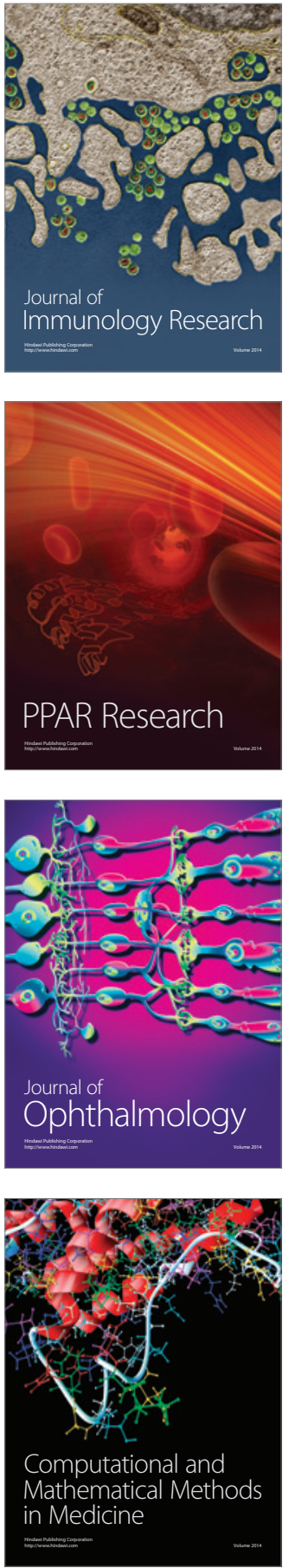

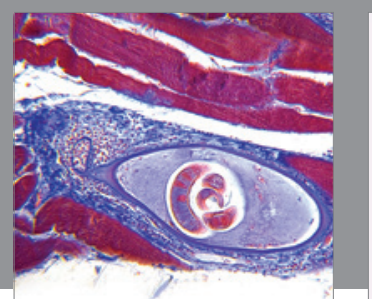

Gastroenterology Research and Practice

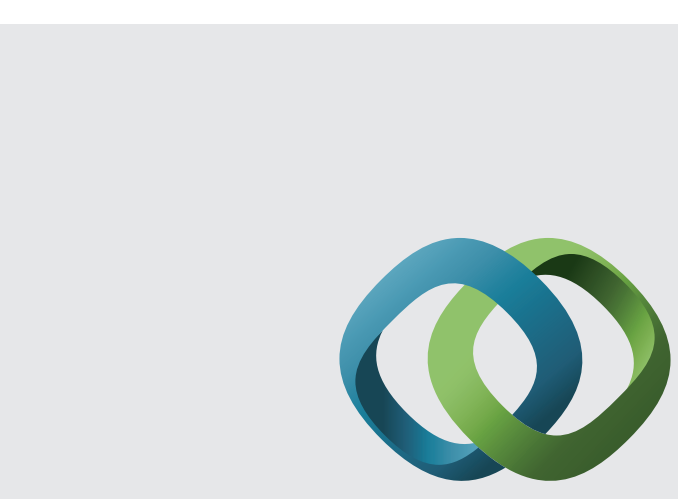

\section{Hindawi}

Submit your manuscripts at

http://www.hindawi.com
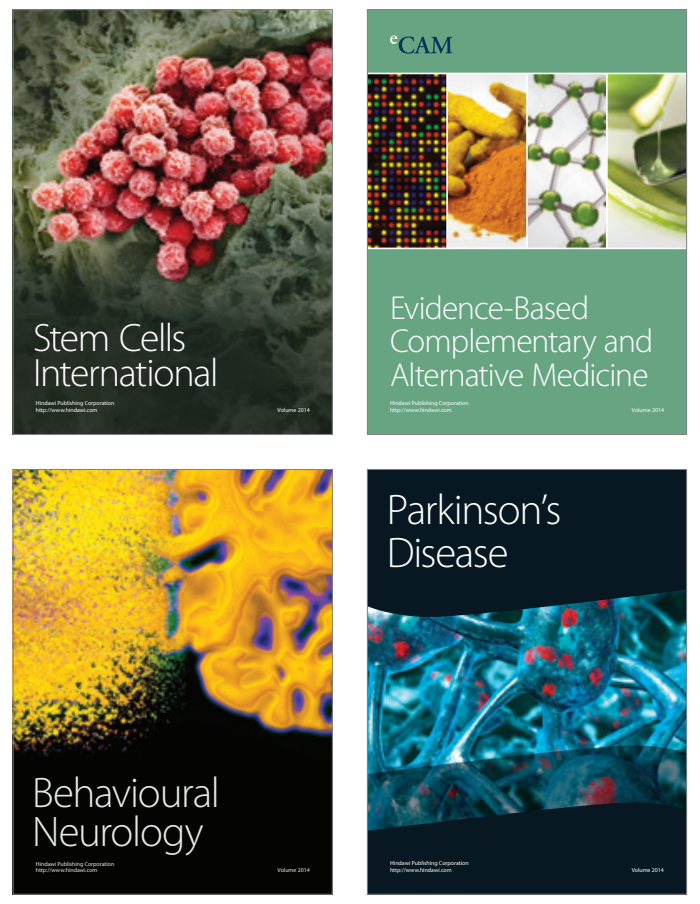
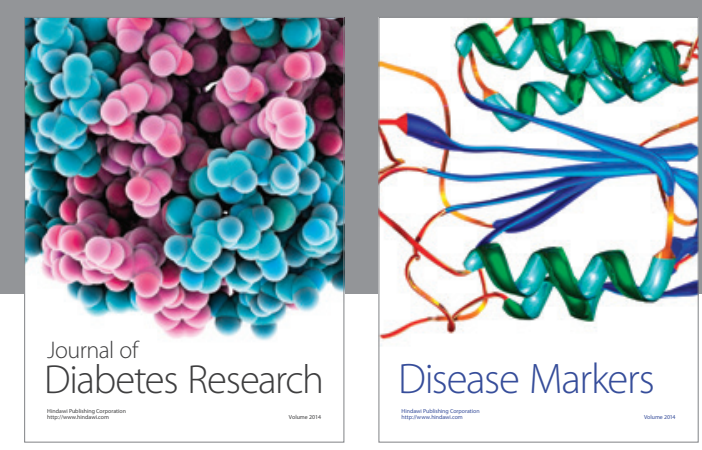

Disease Markers
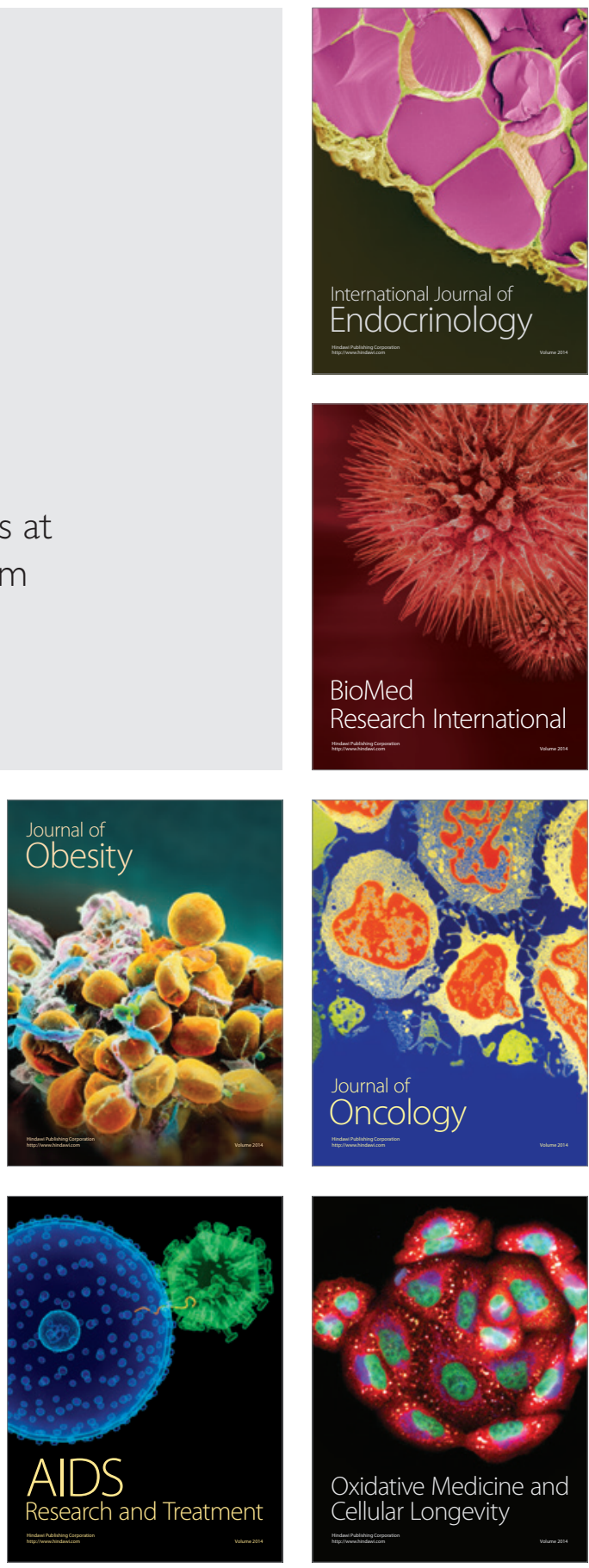